\title{
Autoxidation Products of Polyunsaturated Galactolipids
}

\author{
Ryo Yamauchi, Masahiro KoJima, Koji Kato \\ and Yoshimitsu UENO \\ Department of Agricultural Chemistry, Gifu University, \\ Gifu 501-11, Japan
}

Received June 1, 1983

\begin{abstract}
Neat monogalactosyldilinolenoylglycerol (di-18:3 MGDG) and digalactosyldilinolenoylglycerol (di-18:3 DGDG) were autoxidized at $37^{\circ} \mathrm{C}$. The rate of autoxidation of di-18:3 MGDG was greater than that of di-18:3 DGDG. The oxidation products were separated from unoxidized di18:3 MGDG and di-18:3 DGDG by reverse-phase high performance liquid chromatography (HPLC). The products separated by HPLC were shown to be made up of lipid peroxides formed by free radical oxidation of the linolenate component of the MGDG and DGDG. Each linolenoyl moiety in the MGDG and DGDG produced isomeric peroxides in a manner similar to methyl linolenate.
\end{abstract}

Peroxidation of membrane lipids has been suggested to cause physiological damage in living organisms. In plant, one of the first changes observed when exposed to air pollutants is damage to chloroplast membranes, resulting in a loss of membrane integrity, which is vital to all processes of the plant. ${ }^{1)}$ Isolated chloroplasts, upon illumination, have been reported to undergo destructive peroxidation initiated by light absorbed in chlorophyll. ${ }^{2 \sim 4)}$ The alteration of chloroplast membranes was caused by the peroxidation of unsaturated fatty acids which are constituents of chloroplast membrane lipids. The major lipid classes of chloroplasts are monogalactosyldiacylglycerol (MGDG) and digalactosyldiacylglycerol (DGDG). In higher plants, MGDG and DGDG are characterized by high concentrations of the polyunsaturated fatty acid hexadecatrienoic acid and linolenic acid. ${ }^{5,6)}$ Therefore, the galactolipids were subjected to lipid peroxidation. In our previous study, ${ }^{7)}$ spinach MGDG and DGDG were oxidized with singlet molecular oxygen $\left({ }^{1} \mathrm{O}_{2}\right)$, and mono- and dihydroperoxides formed by ${ }^{1} \mathrm{O}_{2}$ oxidation of the trienoic acid component of MGDG and DGDG were identified.

This paper reports the autoxidation of monogalactosyldilinolenoylglycerol (di-18:3
MGDG) and digalactosyldilinolenoylglycerol (di-18:3 DGDG), which are the major molecular species of spinach galactolipids. ${ }^{8}$ The oxidation products were separated by reversephase high performance liquid chromatography (HPLC) and characterized by gas chromatography-mass spectrometry (GC-MS) after hydrogenation, transmethylation and trimethylsilylation.

\section{MATERIALS AND METHODS}

Materials. MGDG and DGDG were prepared from spinach leaves, and the di-18:3 species were separated by HPLC as described previously. ${ }^{8)}$ Lipase from Rhizopus delemer $(600 \mathrm{U} / \mathrm{mg})$ was obtained from Seikagaku Kogyo Co. Other reagents used were of analytical grade.

Autoxidation procedure. Di-18:3 MGDG and di-18:3 DGDG were autoxidized in a thin lipid film. Fifty $\mu$ of $10^{-2} \mathrm{M}$ lipid in chloroform solution was put into a test tube ( $1.5 \mathrm{~cm}$ in diameter), and then the solvent was removed by evaporation in vacuo to form a thin lipid film. The sample was placed in an incubator maintained at $37^{\circ} \mathrm{C}$ in the dark. At regular intervals, each sample was dissolved in $5 \mathrm{ml}$ of ethanol, and the amount of conjugated diene was determined from the absorbance at $235 \mathrm{~nm}$. For structural analysis, $4 \mathrm{ml}$ of $10^{-2} \mathrm{M}$ lipid solution was put into a round bottom flask $(100 \mathrm{ml})$, and then the solvent was removed by evaporation in vacuo to form a thin lipid film on the inside of the flask. The flask was then maintained at $37^{\circ} \mathrm{C}$ in the dark. 
Characterization of the autoxidized products. The autoxidized MGDG and DGDG were separated by HPLC on a column $(4.6 \times 250 \mathrm{~mm})$ of LiChrosorb RP-18 (Merck, $10 \mu \mathrm{M}$ particle size) using methanol-water $(95: 5, \mathrm{v} / \mathrm{v})$ as the solvent. ${ }^{7)}$ Lipids were detected by refractive index and by absorbance at $235 \mathrm{~nm}$.

The oxidation products separated by HPLC were dissolved in ethanol $(1.0 \mathrm{ml})$ and hydrogenated with palladium on carbon in a stream of hydrogen gas for $1 \mathrm{hr}$. After hydrogenation was completed, the sample was transmethylated with $0.5 \mathrm{M}$ sodium methoxide in methanol solution and converted to trimethylsilyl (TMS) derivatives by heating with TMS-HT/pyridine reagent (Tokyo Kasei Co.). GC-MS analysis was carried out with a Hitachi M52 apparatus equipped with a glass column $(0.3 \times 100 \mathrm{~cm})$ packed with $2 \%$ OV-1 on chromosorb W, $80 \sim 100$ mesh, programmed from $220 \sim 240^{\circ} \mathrm{C}\left(2^{\circ} \mathrm{C} / \mathrm{min}\right)$, the mass spectra being recorded at an ionizing potential of $20 \mathrm{eV}$.

The oxidation products separated by HPLC were hydrolyzed by lipase. The conditions of lipase hydrolysis were the same as described previously. ${ }^{8}$ The resulting fatty acid and oxidized fatty acids were analyzed by thin layer chromatography (TLC) with hexane-ethyl ether-acetic acid $(60: 40: 1, \mathrm{v} / \mathrm{v})$. Spots were detected by spraying with $50 \% \mathrm{H}_{2} \mathrm{SO}_{4}$ followed by charring and the peroxides were detected by spraying with $5 \%$ potassium iodide in methylcellosolve.

\section{RESULTS}

The rate of autoxidation of di-18:3 MGDG and di-18:3 DGDG thin films at $37^{\circ} \mathrm{C}$ is shown in Fig. 1. Both the lipids were immediately oxidized. However the rate of autoxidation of di-18:3 MGDG was greater than that of di-18:3 DGDG.
Di-18:3 MGDG was autoxidized for $5 \mathrm{hr}$ and the oxidation products were analyzed by HPLC. Figure 2 shows the elution pattern of autoxidized di-18:3 MGDG on HPLC. Four new peaks, M1, M2, M3 and M4, were observed in addition to the starting MGDG. The four new peaks were fractionated and their structures were analyzed. M1, M3 and M4, in ethanol, showed an absorption maximum in ultraviolet light at $235 \mathrm{~nm}$, indicating the presence of a conjugated diene, while $\mathbf{M} 2$ showed absorption maxima at 278, 268 and $259 \mathrm{~nm}$, indicating the presence of a conjugated triene.

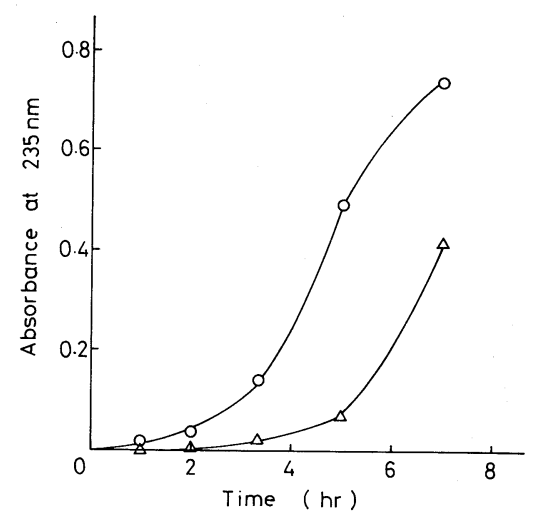

FIG. 1. Time Course of Autoxidation of Di-18:3 MGDG and Di-18:3 DGDG.

Neat di-18:3 MGDG and di-18:3 DGDG were autoxidized at $37^{\circ} \mathrm{C}$. $\bigcirc-\bigcirc$, di-18:3 MGDG; $\triangle-\triangle$, di-18:3 DGDG.
(A)

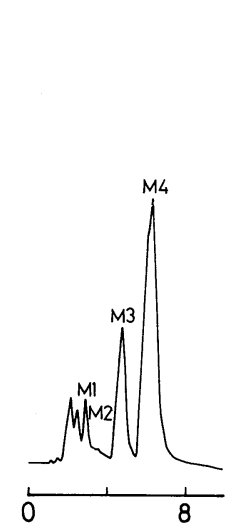

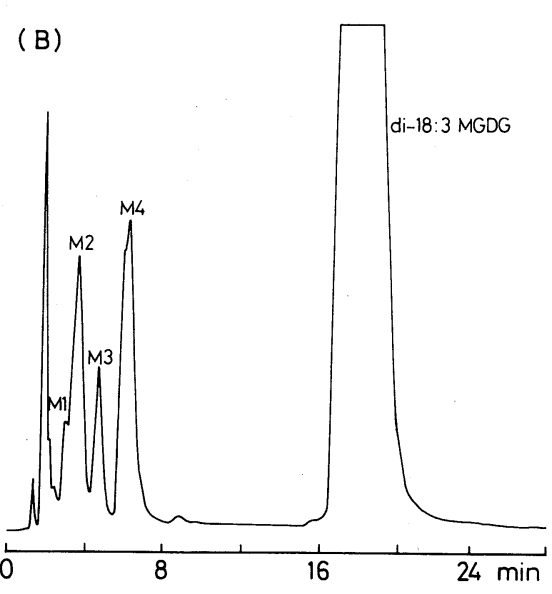

FIG. 2. High Performance Liquid Chromatogram of Autoxidized Di-18:3 MGDG.

Detection was done by absorbance at $235 \mathrm{~nm}$ (A) and by refractive index (B). 
Furthermore, each product was shown to be a peroxide by the potassium iodide test. ${ }^{9)}$ After chemical reduction with triphenylphosphine in ethyl ether solution, M2 and M3 were still found to be peroxides, indicating the presence of endoperoxide. ${ }^{10}$ Therefore, the autoxidation products of di-18:3 MGDG were assumed to be hydroperoxides and endoperoxides.

The products were hydrogenated and transmethylated for GC-MS analysis. Figure 3 shows the gas chromatograms of M1, M2, M3 and M4 after hydrogenation, transmethylation and trimethylsilylation. M1 gave two major peaks, $1 \mathrm{a}$ and $1 \mathrm{~b}$, on the chromatogram (Fig. 3-M1). From the mass spectra, peaks la and $1 \mathrm{~b}$ were identified as methyl hydroxy octadecanoate TMS derivatives $^{11,12)} ; m / z$ 371 [M-

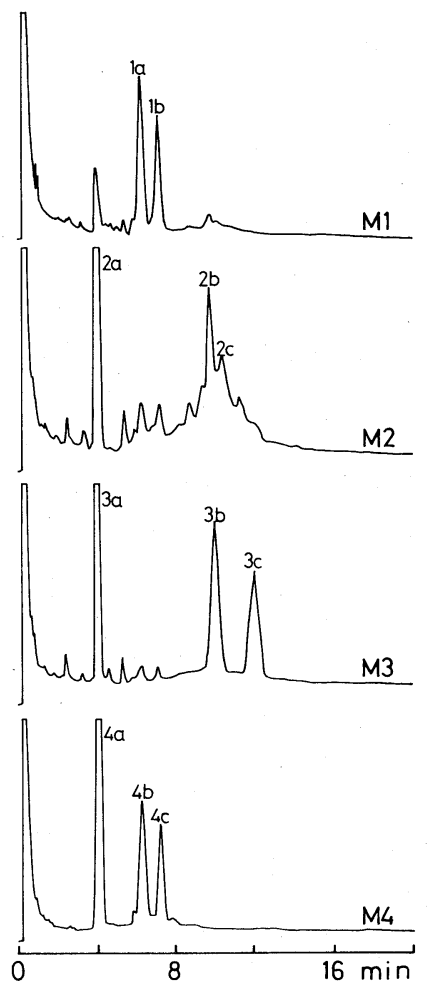

FIG. 3. Gas Chromatograms of M1, M2, M3 and M4 after Hydrogenation, Transmethylation and Trimethylsilylation.

The autoxidized products indicated in Fig. 2 were hydrogenated, transmethylated and silylated for GC-MS analysis. $15]^{+}$and characteristic fragmentation patterns indicated that peak 1a was a mixture of C-9 $(\mathrm{m} / \mathrm{z} 229$ and 259), C-12 ( $\mathrm{m} / \mathrm{z} 187$ and 301) and $\mathrm{C}-13(\mathrm{~m} / \mathrm{z} 173$ and 315$)$ positional isomers, and peak $1 \mathrm{~b}$ was the $\mathrm{C}-16(\mathrm{~m} / \mathrm{z} 131$ and 357$)$ positional isomer of the TMS group, respectively. Therefore, both the linolenoyl moieties of the C-1 and C-2 positions of MGDG were oxidized to form monohydroperoxide isomers.

M2 gave a number of minor components and three major peaks, $2 \mathrm{a}, 2 \mathrm{~b}$ and $2 \mathrm{c}$, on the chromatogram (Fig. 3-M2). Peak 2a was methyl octadecanoate which was derived from the unoxidized linolenoyl moiety of MGDG. Peaks $2 b$ and $2 c$ were analyzed by MS. From the mass spectrum, peak $2 \mathrm{~b}$ was identified as a methyl 9,16-dihydroxy octadecanoate TMS derivatives $^{12)} ; m / z 459[\mathrm{M}-15]^{+}, 445 \alpha$-cleavage of C-16 TMS group, 355 [445-90] ${ }^{+}, 317$ $\alpha$-cleavage of C-9 TMS group, $259 \alpha$-cleavage of C-9 TMS group, 227 [317-90] ${ }^{+}, 159,155$, 137 , and $131 \alpha$-cleavage of C-16 TMS group. The mass spectrum of peak $2 \mathrm{c}$ showed characteristic ions at $m / z \quad 470 \quad[\mathrm{M}-90]^{+}, 455$ $[470-15]^{+}, 439[470-31]^{+}, 380[470-90]^{+}$, 217, $191\left[\left(\mathrm{CH}_{3}\right)_{3}-\mathrm{SiO}=\mathrm{CH}-\mathrm{OSi} \quad\left(\mathrm{CH}_{3}\right)_{3}\right]^{+}$. These fragment ions indicate that peak $2 \mathrm{c}$ contains a silylated dihydroxy cyclopentane ring. ${ }^{13)}$ Ions due to $\alpha$-cleavage of the TMS group were assigned to every position in the hydroxy group as follows: C-9 $(\mathrm{m} / \mathrm{z} 259,313$ $\left.[403-90]^{+}, 223[313-90]^{+}\right), \mathrm{C}-11(\mathrm{~m} / z$ 287,

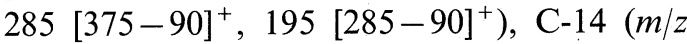
$\left.159,413[503-90]^{+}\right)$, and C-16 $(m / z 131,441$ $\left.[531-90]^{+}, 351[441-90]^{+}\right)$. Thus, it is apparent that peak $2 \mathrm{c}$ contains trihydroxy compounds consisting of the 9,13,15-, 11,13,15-, 10,12,14- and 10,12,16-trihydroxy regioisomers. These trihydroxy compounds were reported to be derivatives of hydroperoxy bicyclic endoperoxide which were produced on the peroxidation of methyl linolenate. ${ }^{14 \sim 17)}$

M3 gave three peaks, 3a, 3b and 3c, on the chromatogram (Fig. 3-M3). Peak 3a was methyl octadecanoate. Peaks $3 b$ and $3 c$ were analyzed by MS. From the mass fragmentation patterns of their mass spectra, ${ }^{11,12)}$ peak $3 \mathrm{~b}$ was identified as a methyl 9,10,12-trihy- 
droxy octadecanoate TMS derivative; $m / z 457$ $[\mathrm{M}-(90+15)]^{+}, 441[\mathrm{M}-(90+31)]^{+}, 387$, $332,259,187$ and 155, and peak $3 \mathrm{c}$ was identified as a methyl 13,15,16-trihydroxy octadecanoate TMS derivative; $m / z \quad 457$ $[\mathrm{M}-(90+15)]^{+}, 441[\mathrm{M}-(90+31)]^{+}, 341$, $315,259,233,211,169,143$ and 131. These trihydroxy compounds can be produced by hydrogenation of hydroperoxy 5-membered endoperoxides. ${ }^{10,17 \sim 19)}$ Thus, it is apparent that M3 contains an isomeric mixture of hydroperoxy endoperoxides.

M4 gave three peaks, 4a, 4b and 4c, on the chromatogram (Fig. 3-M4). Peak 4a was methyl octadecanoate. Peaks $4 \mathrm{~b}$ and $4 \mathrm{c}$ were methylhydroxy octadecanoate TMS derivatives as judged from their mass spectra. The isomer distribution of the products was the same as that of M1.

The peak area corresponding to methyl octadecanoate as shown in Fig. 3 comprised about $50 \%$ of the total area in every chromatogram of M2, M3 and M4. These observations indicate that the linolenoyl moiety of either the C- 1 or $\mathrm{C}-2$ position of MGDG is oxidized and the other moiety is unoxidized. The fractions

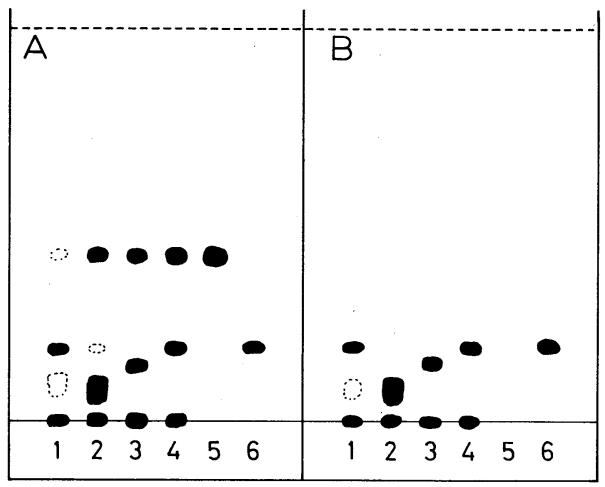

FIG. 4. Thin Layer Chromatogram of Lipase Hydrolysis Products of Autoxidized Di-18:3 MGDG.

The autoxidized products, M1, M2, M3 and M4, were hydrolyzed by lipase.

The resulting products of M1 (1), M2 (2), M3 (3) and M4 (4), linolenic acid (5) and linolenic acid monohydroperoxide (6) were charged on the TLC plate and developed with hexane-ethyl ether-acetic acid $(60: 40: 1, \mathrm{v} / \mathrm{v})$. Spots were detected by spraying with $50 \% \mathrm{H}_{2} \mathrm{SO}_{4}$ followed by charring (A) or by spraying with $5 \%$ potassium iodide solution (B). of autoxidized di-18:3 MGDG as shown in Fig. 2 were hydrolyzed by Rhizopus delemer lipase which hydrolyzed the C-1 position fatty acid of galactolipids. ${ }^{20)}$ The resulting products were analyzed by TLC. Figure 4 shows the hydrolysis products of M1, M2, M3 and M4 on the TLC plate. Spots corresponding to fatty acid and oxidized fatty acids appeared on the plate. Spots corresponding to oxidized fatty acids turned yellow on spraying with $5 \%$ potassium iodide solution, indicating the presence of peroxide. ${ }^{9)}$ Thus, it seems likely that oxidized fatty acid components of MGDG are located at both the $\mathrm{C}-1$ and $\mathrm{C}-2$ positions of MGDG.

From the above data, the compound in each peak indicated in Fig. 2 was characterized to be as follows: M1, 1,2-dihydroperoxyoctadecatrienoyl MGDG; M2, the linolenoyl moiety of either the $\mathrm{C}-1$ or $\mathrm{C}-2$ position of MGDG was oxidized to form 9,16-dihydroperoxide and hydroperoxy bicyclic endoperoxides and the other moiety was unoxidized; M3, the linolenoyl moiety of either the $\mathrm{C}-1$ or $\mathrm{C}-2$ position of MGDG was oxidized to form hydroperoxy endoperoxides and the other moiety was unoxidized; M4, 1-linolenoyl-2hydroperoxyoctadecatrienoyl MGDG and 1hydroperoxyoctadecatrienoyl-2-linolenoyl MGDG.
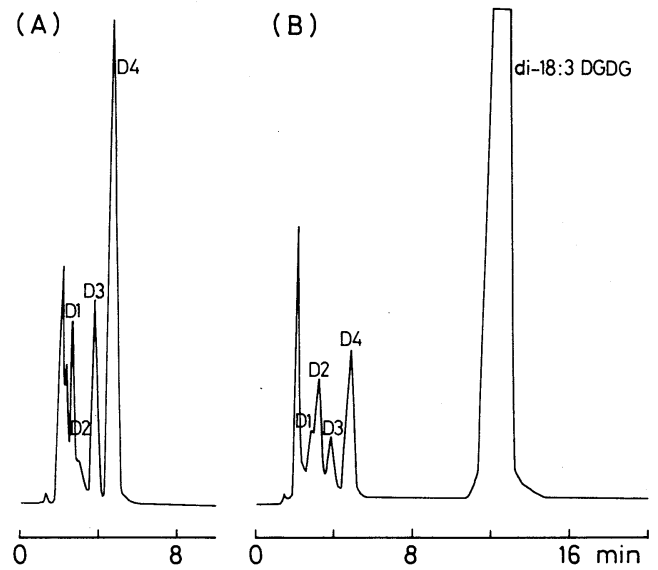

FIG. 5. High Performance Liquid Chromatogram of Autoxidized Di-18:3 DGDG.

Detection was done by absorbance at $235 \mathrm{~nm}$ (A) and by refractive index (B). 
Di-18:3 DGDG was also autoxidized for $8 \mathrm{hr}$ and then analyzed by HPLC (Fig. 5). Four new peaks, D1, D2, D3 and D4, were observed in addition to the starting DGDG. The four peaks were fractionated to analyze their structures. D1, D3 and D4, in ethanol, had an absorption maximum in ultraviolet light at $235 \mathrm{~nm}$, while D2 showed absorption maxima at 278,268 and $259 \mathrm{~nm}$. These products were shown to be peroxides. After reduction with triphenylphosphine, D2 and D3 were still found to be peroxides. From the GC-MS analysis after hydrogenation, transmethylation and trimethylsilylation and the TLC analysis of the hydrolyzed products by lipase, the structures of D1, D2, D3 and D4 corresponded to those of M1, M2, M3 and M4, respectively, that is: D1, 1,2-dihydroperoxyoctadecatrienoyl DGDG; D2, the linolenoyl moiety of either the $\mathrm{C}-1$ or $\mathrm{C}-2$ position of DGDG was oxidized to form 9,16-dihydroperoxide and hydroperoxy bicyclic endoperoxides and the other moiety was unoxidized; D3, the linolenoyl moiety of either the C-1 or C-2 position of DGDG was oxidized to form hydroperoxy endoperoxides and the other moiety was unoxidized; D4, 1-linolenoyl-2hydroperoxyoctadecatrienoyl DGDG and 1hydroperoxyoctadecatrienoyl-2-linolenoyl DGDG.

\section{DISCUSSION}

The autoxidation of neat di-18:3 MGDG and di-18: 3 DGDG at $37^{\circ} \mathrm{C}$ is reasonably fast. The rate of oxidation of di-18:3 MGDG was found to be greater than that of di-18:3 DGDG. The acyl moieties of MGDG and DGDG were the same, linolenate, and the difference between the two lipids was the number of galactose moieties. Corliss and Dugan, $\mathrm{Jr}^{21}{ }^{21}$ have reported that the nitrogen moieties, ethanolamine and choline, influenced the induction period for the oxidation of phosphatidylethanolamine and phosphatidylcholine, respectively. Furthermore, non-reducing sugars have been reported to inhibit the autoxidation of methyl linoleate. ${ }^{22)}$
Therefore, the galactose moieties of galactolipids may influence the oxidation rate of MGDG and DGDG.

Autoxidation of di-18:3 MGDG and di18:3 DGDG seems capable of forming many oxygenated compounds (Figs. 2 and 5). The MGDG and DGDG were found to be autoxidized at either the C-1 or C-2 position of the linolenoyl moiety to produce monohydroperoxides, hydroperoxy endoperoxides, hydroperoxy bicyclic endoperoxides and a dihydroperoxide. Both the C-1 and C-2 positions of linolenoyl moieties could also be oxidized to produce 1,2dihydroperoxy linolenoyl compounds. Polyunsaturated fatty acid and esters are particularly liable to undergo air oxidation by a free radical chain mechanism. Many workers have reported the free radical oxidation of methyl linolenate and have characterized many oxidized products, monohydroperoxide isomers,${ }^{11,12,23)}$ a dihydroperoxide ${ }^{10,12)}$ hydroperoxy endoperoxide isomers, ${ }^{10 \sim 12)}$ and hydroperoxy bicyclic endoperoxide isomers. ${ }^{15)}$ It appears that each linolenoyl moiety comprising MGDG and DGDG produced isomeric monohydroperoxides in a manner similar to methyl linolenate. Thus, 9-, 12-, 13- and 16-isomers were detected. The linolenoyl moiety of MGDG and DGDG was also further oxidized in a manner similar to methyl linolenate.

Plant galactolipids are readily oxidized by ${ }^{1} \mathrm{O}_{2}$ and by autoxidation. Despite many catalytic metal complexes and photosensitizers found in plants, plant chloroplast membranes have a mechanism for protection against peroxidation. Therefore, research on natural antioxidants, such as $\alpha$-tocopherol, ${ }^{4)}$ and ${ }^{1} \mathrm{O}_{2}$ quenchers, such as $\beta$-carotene, ${ }^{24)}$ may unravel the mechanism plants have for protection against lipid peroxidation.

\section{REFERENCES}

1) G. D. Peiser, Ma. C. C. Lizada and S. F. Yang, Plant Physiol., 70, 994 (1982).

2) R. L. Heath and L. Packer, Arch. Biochem. Biophys., 125, 189 (1968); R. L. Heath and L. Packer, Arch. Biochem. Biophys., 125, 850 (1968). 
3) U. Takahama and M. Nishimura, Plant Cell Physiol., 16, 737 (1975); U. Takahama and M. Nishimura, Plant Cell Physiol., 17, 111 (1976).

4) R. Yamauchi and S. Matsushita, Agric. Biol. Chem., 43, 2157 (1979).

5) C. F. Allen, P. Good, H. F. Davis, P. Chisum and S. D. Fowler, J. Am. Oil Chem. Soc., 43, 223 (1966).

6) M. Nishihara, K. Yokota and M. Kito, Biochim. Biophys. Acta, 617, 12 (1980).

7) R. Yamauchi, M. Kojima, M. Isogai, K. Kato and Y. Ueno, Agric. Biol. Chem., 46, 2815 (1982).

8) R. Yamauchi, M. Kojima, M. Isogai, K. Kato and Y. Ueno, Agric. Biol. Chem., 46, 2847 (1982).

9) K. Oette, J. Lipid Res., 6, 449 (1965).

10) W. E. Neff, E. N. Frankel and D. Weisleder, Lipids, 16, 439 (1981).

11) E. N. Frankel, W. E. Neff, W. K. Rohwedder, B. P. S. Khambay, R. F. Garwood and B. C. L. Weedon, Lipids, 12, 1055 (1977).

12) I. Toyoda, J. Terao and S. Matsushita, Lipids, 17, 84 (1982).

13) P. Farardeau, M. Hamberg and B. Samuelsson, Biochim. Biophys. Acta, 441, 193 (1976).
14) W. A. Pryor and J. P. Stanley, J. Org. Chem., 40, 3615 (1975).

15) W. A. Pryor, J. P. Stanley and E. Blair, Lipids, 11, 370 (1976).

16) D. E. O'Connor, E. D. Mihelich and M. C. Coleman, J. Am. Chem. Soc., 103, 223 (1981).

17) W. E. Neff, E. N. Frankel and D. Weisleder, Lipids, 17, 780 (1982)

18) D. T. Coxon, K. R. Price and H. W.-S. Chan, Chem. Phys. Lipids, 28, 365 (1981).

19) E. N. Frankel, W. E. Neff, E. Selke and D. Weisleder, Lipids, 17, 11 (1982).

20) W. Fisher, E. Heinz and M. Zeus, Hoppe-Seyler's Z. Physiol. Chem., 354, 1115 (1973).

21) G. A. Corliss and L. R. Dugan, Jr., Lipids, 5, 846 (1970).

22) R. Yamauchi, Y. Aoki, T. Sugiura, K. Kato and Y. Ueno, Agric. Biol. Chem., 46, 2997 (1982).

23) E. N. Frankel, C. D. Evance, D. G. McConnel, E. Selke and H. J. Dutton, J. Org. Chem., 26, 4663 (1961).

24) C. S. Foote, Y. C. Chang and R. W. Denny, J. Am. Chem. Soc., 92, 5216 (1970). 